\title{
Papel do cuidador domiciliar na assistência ao paciente portador do transtorno do espectro autista: Uma revisão integrativa.
}

Role of home caregivers in assisting patients with autism spectrum disorder: An integrative review. Papel de los cuidadores domiciliariosenlaatención de pacientes contrastornosdel espectro autista: Una revisión integradora.

Allan Bruno Alves de Sousa Santos ORCID: https://orcid.org/0000-0001-6412-7164

Faculdade de Educação São Francisco, Brasil E-mail: abass@faesf.com.br

Débora de Oliveira Lima

ORCID: https://orcid.org/0000-0002-8706-9451

Universidade Federal do Piauí, Brasil

E-mail: deboralima209@gmail.com

Ana Carolina do Nascimento Simões da Silva

ORCID: https://orcid.org/0000-0002-3905-6621

Universidade Augusto Motta, Brasil

E-mail: Acarolina2205@gmail.com

Kallyne dos Santos

ORCID: https://orcid.org/0000-0002-1938-0539

Centro Universitário Integrado, Brasil

E-mail: kallynesant@outlook.com

Mikaelle Alves Silva

ORCID: https://orcid.org/0000-0002-9336-1491

Universidade Federal do Espírito Santo, Brasil

E-mail: farm.mikaelle@gmail.com

Paloma Vitória Menezes dos Santos

ORCID: https://orcid.org/0000-0001-9513-9846

Faculdade de Ciências e Empreendedorismo, Brasil

E-mail: paloma_vitoriams@hotmail.com

Carla Conrado Barbosa

ORCID: https://orcid.org/0000-0002-7033-3682

Faculdade da Polícia Militar, Brasil

E-mail: carlaconradobl@gmail.com

Douglas Bento Chagas

ORCID: https://orcid.org/0000-0003-3141-8840 Universidade Federal de Pelotas, Brasil

E-mail: douglasbchagas@hotmail.com

Luíza Alves da Silva

ORCID: https://orcid.org/0000-0002-4497-841X

Universidade Federal do Piauí, Brasil

E-mail: luizaalves.silva83@gmail.com

Luciana Carneiro de Brito Ramalho

ORCID: https://orcid.org/0000-0002-4258-8143 Faculdade Bezerra de Araújo, Brasil

E-mail: luciana016@gmail.com

Bianca Barroso de Sousa

ORCID: https://orcid.org/0000-0002-3521-6667

Universidade Estadual do Maranhão, Brasil

E-mail: biancabarroso000@gmail.com

Leandro Barbosa Teixeira

ORCID: https://orcid.org/0000-0002-9444-7828

Universidade Estácio de Sá, Brasil

E-mail: Lbtteixeira@ hotmail.com

Dalton Ferreira Matos

ORCID: https://orcid.org/0000-0002-6188-7536

Universidade Federal de Sergipe, Brasil

E-mail: daltonmatosgn@gmail.com

Lorrayne Valério de Sá

ORCID: https://orcid.org/0000-0002-7880-2279

Universidade Federal Fluminense, Brasil

E-mail: lovalerio@id.uff.br 
Valéria Fernandes da Silva Lima

ORCID: https://orcid.org/0000-0002-7516-4806

Universidade Estadual do Maranhão, Brasil

E-mail: valeriafernandesxp@gmail.com

Juliana da Silva Sousa

ORCID: https://orcid.org/0000-0002-5653-9473 Christus Faculdade do Piauí, Brasi E-mail: enfjuh22@gmail.com

Maria Eduarda de Barros Pardelhas

ORCID: https://orcid.org/0000-0001-6881-1959 Universidade Federal do Estado do Rio de Janeiro, Brasil E-mail: mepardelhas@edu.unirio.br

Maria Helena de Lima Sousa

ORCID: https://orcid.org/0000-0003-1866-8618

Faculdade de Tecnologia e Ciências, Brasil

E-mail: helenamaria164@gmail.com

Maria Samara da Silva

ORCID: https://orcid.org/0000-0001-6987-1224 Universidade Estadual do Piauí, Brasil

E-mail: mariasamara2v@gmail.com

Gabriela Cristina Limp

ORCID: https://orcid.org/0000-0002-3911-3948

Centro Universitário Augusto Motta, Brasil

E-mail: limpgabriela@gmail.com

Luani Akemi Furyama

ORCID: https://orcid.org/0000-0002-5472-5280

Universidade Paranaense, Brasil

E-mail: luani.furyama@gmail.com

Laiany Maiara Quirino de Paula

ORCID: https://orcid.org/0000-0002-3375-6029

Universidade Paranaense, Brasil

E-mail: 1.paula@edu.unipar.br

Nair Arrais Leite

ORCID: https://orcid.org/0000-0003-2773-9350

Faculdade de Educação São Francisco, Brasi E-mail: nal@ faesf.com.br

Elias Paulo da Silva

ORCID: https://orcid.org/0000-0001-7583-7567 Universidade Federal do Piauí, Brasil E-mail: eliaspaulo.silva@gmail.com

Islaila Maria Silva Ferreira

ORCID: https://orcid.org/0000-0002-5736-883X Universidade Federal do Piauí, Brasil E-mail: islailamsf28@gmail.com

Gedelvani Francisco Oliveira da Silva

ORCID: https://orcid.org/0000-0002-3591-1408 Christus Faculdade do Piauí, Brasil E-mail: gedelvaniolivera@gmail.com

\section{Resumo}

Este estudo tem como objetivo buscar na literatura as atividades realizadas pelo cuidador familiar a pessoa portadora do transtorno do espectro autista, apontando as suas principais dificuldades. E tem a importância de estimular a pesquisa nessa temática, abordando com ênfase ação dos cuidadores no domicilio. Trata-se de um estudo do tipo revisão integrativa de caráter qualitativo, a busca ocorreu nas bases de dados: Literatura Latino-Americana e do Caribe em Ciências da Saúde (LILACS); ScientificElectronic Library Online (SciELO) e Base de Dados de Enfermagem (BDENF). Os artigos foram coletados nos períodos de junho a julho de 2021. Foram utilizados os descritores: "Cuidadores; Assistência Domiciliar e Transtorno do Espectro Autista", selecionados na lista dos Descritores em Ciências da Saúde (DECs). Foram selecionados os critérios para exclusão e inclusão no estudo, e restaram um quantitativo de 9 pesquisas usadas. A principal atividade desempenhada no cuidado a pacientes com TEA, cabe a responsabilidade das cuidadoras, sendo em sua maioria compostas por mães de pacientes, os cuidadores do sexo masculino, desempenha atividades conceituadas como auxiliares, o que gera uma sobrecarga de trabalho as cuidadoras familiares. Conclui-se que é necessário promover saúde a esses cuidadores, pois ficou claro durante a construção do estudo, que essa comunidade ainda é carente de informações sobre o diagnóstico e tratamento de TEA.

Palavras-chave: Cuidadores; Assistência domiciliar; Transtorno do espectro autista.

\section{Abstract}

This study aims to search the literature for activities performed by family caregivers to people with autism spectrum disorder, pointing out their main difficulties. And it is important to encourage research on this theme, emphasizing the 
action of caregivers at home. This is an integrative review study of a qualitative nature, the search was carried out in the following databases: Latin American and Caribbean Literature in Health Sciences (LILACS); ScientificElectronic Library Online (SciELO) and Nursing Database (BDENF). The articles were collected from June to July 2021. The descriptors were used: "Caregivers; Home Care and Autistic Spectrum Disorder", selected from the list of Health Sciences Descriptors (DECs). Criteria for exclusion and inclusion in the study were selected, leaving a quantitative of 9 researches used. The main activity performed in the care of patients with ASD is the responsibility of caregivers, being mostly composed of mothers of patients, male caregivers, performing activities regarded as assistants, which generates a work overload for family caregivers. It is concluded that it is necessary to promote health to these caregivers, as it was clear during the construction of the study that this community still lacks information about the diagnosis and treatment of ASD.

Keywords: Caregivers; Home care; Autistic spectrum disorder.

\section{Resumen}

Este estudio tiene como objetivo buscar en la literatura las actividades que realizan los cuidadores familiares a personas con trastorno del espectro autista, señalando sus principales dificultades. Y es importante fomentar la investigación sobre este tema, haciendo hincapié en la acción de los cuidadores en el hogar. Se trata de un estudio de revisión integradora de carácter cualitativo, la búsqueda se realizó en las siguientes bases de datos: Literatura Latinoamericana y del Caribe en Ciencias de la Salud (LILACS); Biblioteca Electrónica Científica en Línea (SciELO) y Base de Datos de Enfermería (BDENF). Los artículos fueron recolectados de junio a julio de 2021. Se utilizaron los descriptores: "Cuidadores; Atención domiciliaria y trastorno del espectro autista", seleccionado de la lista de descriptores de ciencias de la salud (DEC). Se seleccionaron los criterios de exclusión e inclusión en el estudio, y aún se utilizaron 9 investigaciones para la construcción del estudio. La principal actividad que se realiza en el cuidado de pacientes con TEA es responsabilidad de los cuidadores, estando mayoritariamente compuesta por madres de pacientes, cuidadores masculinos, realizando actividades consideradas como asistentes, lo que genera una sobrecarga de trabajo para los cuidadores familiares. Se concluye que es necesario promover la salud de estos cuidadores, pues quedó claro durante la construcción del estudio que esta comunidad aún carece de información sobre el diagnóstico y tratamiento de los TEA.

Palabras clave: Cuidadores; Atención domiciliaria; Trastorno del espectro autista.

\section{Introdução}

Segundo a Organização Pan- Americana de saúde (OPAS), em relação ao panorama mundial, 1 a cada 160 crianças, nasce com o transtorno do espectro autista (TEA), e o número de casos se caracteriza crescente nos últimos 50 anos, sendo atribuída a um dos principais motivos, a informação disseminada na sociedade e um programa de captação e diagnósticos aprimorado (Organização pan-americana de saúde, 2020).

No panorama nacional, segundo o Ministério da Saúde, estima-se que dos 200 milhões de habitantes, há cerca de 2 milhões de autistas no Brasil, sendo a região centro-oeste a primeira em taxas de ocorrências. E apesar das altas taxas, o autismo por muitos anos foi negligenciado e ignorado em vários países do mundo, sendo apenas no ano de 1993 , que a patologia foi adicionada a Classificação internacional de doenças feita pela organização mundial da saúde (Oliveira, 2020).

O transtorno do Espectro Autista é conceituado como um conjunto de distúrbios do neurodesenvolvimento, enfatizado por uma diminuição na percepção, interação social e deficiência intelectual, e sua sintomatologia começa em média nos cinco primeiros anos de vida e perdura pelo resto da mesma (Ministério da Saúde,2014).

O diagnóstico é facilitado quando os sinais de diminuição de reflexos são apresentados ainda nas fases iniciais de vida, tais como acompanhar objetos com os olhos, agarrar objetos e exercer comportamento de reconhecimento fácil, todas essas características são diminuídas na criança com TEA (Ministério da saúde, 2014).

O instrumento de rastreamento usado pelas equipes de saúde no âmbito do Sistema Único de Saúde (SUS) é fator importante para o diagnóstico precoce, e sendo assim exclusivamente clinico, necessitando de o profissional habilidades de reconhecimento de fatores predisponentes ao TEA, sendo de fundamental importância o uso de tecnologias leves de cuidado, enfatizando o acolhimento e escuta qualificada, no qual através do diálogo com a família e observando o paciente, o profissional vai realizar o diagnóstico, e a triagem pode ser aplicada pela equipe multiprofissional, e as ferramentas de rastreio é disponibilizada pelo serviço de saúde (Ministério da saúde, 2014). 
O TEA retarda em proporções significativas a capacidade de o indivíduo exercer suas atividades cotidianas, e sofrer com a exclusão nas escolas e oportunidades empregatícias, e no domicilio necessita em casos mais graves, de um cuidador, alguém responsável por auxiliar no desenvolvimento das atividades básicas de vida e aplicar tarefas estimulantes do sistema nervoso central, para promover efeitos terapêuticos ao paciente (Organização pan-americana de saúde, 2020).

$\mathrm{O}$ estudo aponta o seguinte objetivo: Buscar na literatura as atividades realizadas pelo cuidador familiar a pessoa portadora do transtorno do espectro autista, apontando as suas principais dificuldades. E como proposta a referente pesquisa almeja estimular os estudos nessa temática, abordando com ênfase ação dos cuidadores no domicilio.

\section{Metodologia}

Trata-se de uma revisão do tipo integrativa de caráter qualitativo. Segundo Ercole, Melo \& Alcoforado (2014), esse tipo de revisão é utilizado para sintetização de resultados obtidos através de uma determinada pesquisa nas bases de dados, sobre uma temática especifica e se caracterizando por ser sistemático.

Foi estabelecido as seguintes etapas para a pesquisa:1) Definição da temática e pergunta norteadora através da estratégia PIco; 2) Elaboração dos critérios de inclusão e exclusão para o estudo; 3) Definição das bases de dados e descritores;4) Busca do material para desenvolvimento da pesquisa; 5) Analise crítica e discussão dos resultados.

Para nortear o estudo definiu-se a seguinte questão: "O que a literatura aborda sobre as atividades realizadas pelo cuidador familiar a pessoa portadora do transtorno do espectro autista?".

Para a elaboração do estudo, a coleta de dados foi realizada na base de dados Literatura Latino-Americana e do Caribe em Ciências da Saúde (LILACS); ScientificElectronic Library Online (SciELO) e Base de Dados de Enfermagem (BDENF). Foram utilizados os descritores "Cuidadores; Assistência Domiciliar e Transtorno do Espectro Autista", selecionados na lista dos Descritores em Ciências da Saúde (DECs), e cruzados com o opbooleano "AND" e "OR". A pesquisa ocorreu no período de junho a julho de 2021. A estratégia para elaboração do tema e questão norteadora foi a PIco, identificando a população, intervenção e o contexto do estudo.

Foram selecionados os critérios para exclusão no estudo: Artigos incompletos, duplicados, resumos, teses, dissertações, com recorte temporal inferior a 2014 e que não abordassem o objetivo da pesquisa. Os critérios para inclusão no estudo abordaram artigos completos disponíveis na integra, com recorte temporal de 2014 a 2021, na qual foi estendido o recorte temporal, pois encontra-se poucas pesquisas acerca da temática, e estudos em idioma português e inglês e que respondessem à pergunta norteadora da pesquisa.

\section{Resultados e Discussão}

No fluxograma a seguir apresenta toda a estratégia de busca, detalhando as principais etapas para construção do estudo. 
Figura 1. Fluxograma com a trajetória da seleção dos manuscritos.

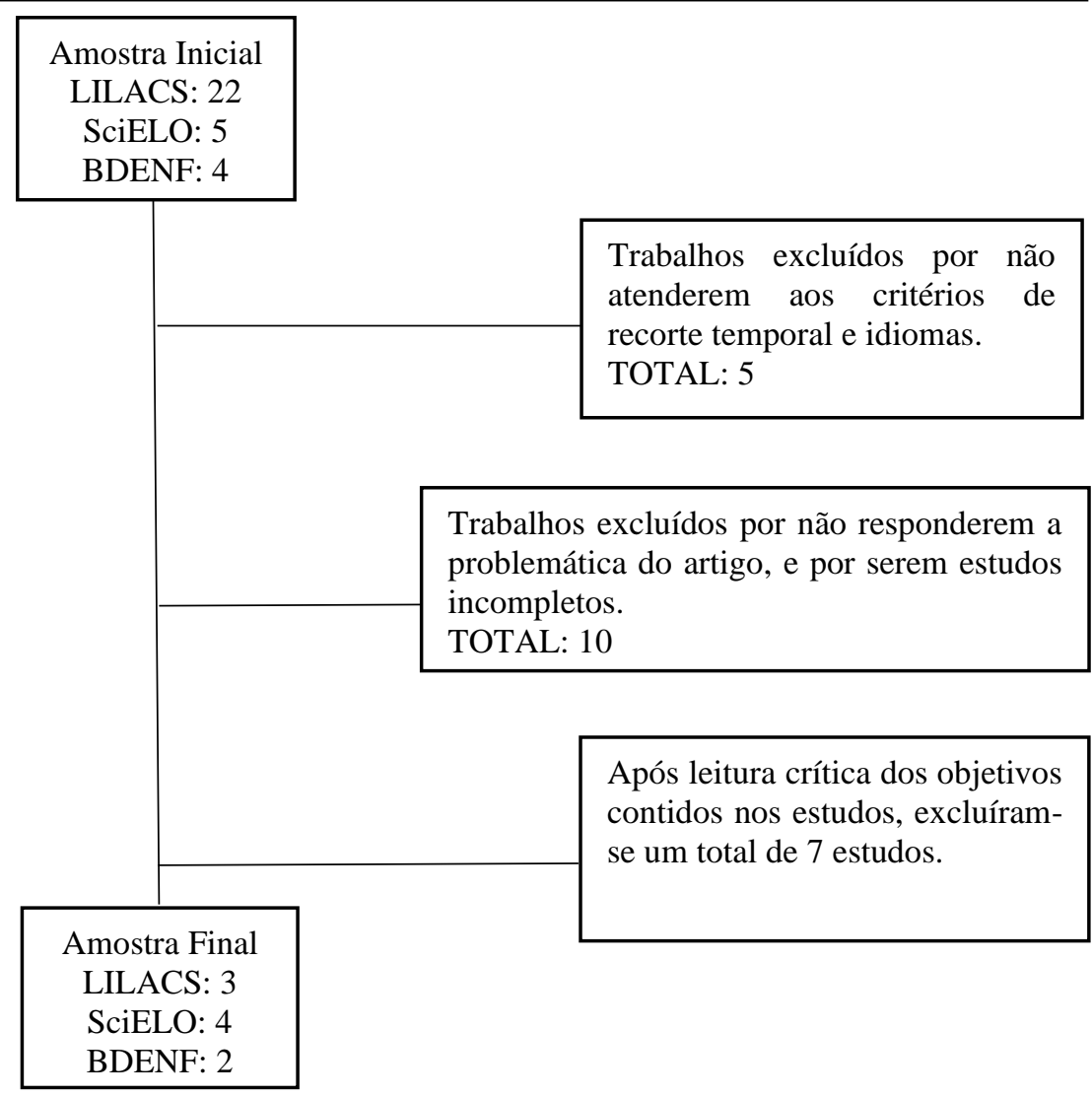

Fonte: Autores (2021).

No Fluxograma 1, observa-se que na amostra inicial da coleta, constatou-se 31 estudos, que foram submetidos a primeira etapa de avaliação por meio dos critérios de exclusão e inclusão. Assim, após aplicar os critérios de recorte temporal e idioma, excluíram-se 5 estudos. Os estudos foram submetidos a leitura e analise critica, e excluíram-se mais 10 estudos por não retratar a temática da pesquisa.

Na segunda etapa, feito a leitura dos manuscritos, observou-se um quantitativo de 9 estudos que abordavam a temática e respondiam à questão norteadora da pesquisa.

O Quadro 1, a seguir, apresenta os estudos selecionados para a pesquisa segundo sua autoria, título, método e ano. 
Quadro 1. Artigos referentes ao estudo.

\begin{tabular}{|c|c|c|c|}
\hline Autor & Título & Método & Ano \\
\hline $\begin{array}{l}\text { Magagninet } \\
\text { al. }\end{array}$ & $\begin{array}{l}\text { Aspectos alimentares e nutricionais decrianças e } \\
\text { adolescentes com transtorno doespectro autista. }\end{array}$ & $\begin{array}{l}\text { Estudo exploratório e descritivo de } \\
\text { aspecto qualitativo. }\end{array}$ & 2021 \\
\hline $\begin{array}{l}\text { Moreira, } \\
\text { Lima } \\
\text { \&Guerra. }\end{array}$ & $\begin{array}{l}\text { Sobrecarga do cuidador informal de crianças com } \\
\text { transtorno do espectrodo autista. }\end{array}$ & Estudo descritivo-correlacional. & 2020 \\
\hline $\begin{array}{l}\text { Portes } \quad \& \\
\text { Vieira. }\end{array}$ & $\begin{array}{l}\text { Coparentalidade no contexto familiar de crianças com } \\
\text { transtorno do espectro autista. }\end{array}$ & $\begin{array}{l}\text { Estudo exploratório e descritivo de } \\
\text { abordagem qualitativo. }\end{array}$ & 2020 \\
\hline $\begin{array}{l}\text { Bagaioloet } \\
\text { al. }\end{array}$ & $\begin{array}{l}\text { Implementing a community-basedparent training } \\
\text { behavioralintervention for Autism Spectrum Disorder. }\end{array}$ & Estudo não controlado e multicêntrico. & 2019 \\
\hline $\begin{array}{l}\text { Fonseca et } \\
\text { al. }\end{array}$ & $\begin{array}{l}\text { Influências do transtorno do espectroautista nas relações } \\
\text { familiares: revisão sistemática. }\end{array}$ & ica da literatura. & 2019 \\
\hline $\begin{array}{l}\text { Balestro\& } \\
\text { Fernandes. }\end{array}$ & $\begin{array}{l}\text { Percepção de cuidadores de crianças com Transtorno do } \\
\text { Espectro do Autismo quanto ao perfil comunicativo de } \\
\text { seus filhos após um programa de orientação } \\
\text { fonoaudiológica. }\end{array}$ & Estudo exploratório e descritivo. & 2018 \\
\hline $\begin{array}{ll}\text { Corrêa } & \text { e } \\
\text { Queiroz. } & \end{array}$ & $\begin{array}{l}\text { A família é o melhor recurso da criança: análise das } \\
\text { trocas sociais entre mães e crianças com transtorno do } \\
\text { espectro do autismo. }\end{array}$ & $\begin{array}{l}\text { Estudo descritivo de abordagem } \\
\text { qualitativa. }\end{array}$ & 2017 \\
\hline $\begin{array}{l}\text { Tabaquimet } \\
\text { al. }\end{array}$ & $\begin{array}{l}\text { Autoeficácia de cuidadores de criançascom o transtorno } \\
\text { do espectro autista. }\end{array}$ & $\begin{array}{l}\text { Estudo exploratório e descritivo de } \\
\text { aspecto qualitativo. }\end{array}$ & 2015 \\
\hline Gomes et al. & $\begin{array}{l}\text { Autism in Brazil: a systematic } \\
\text { offamilychallengesandcopingstrategies. }\end{array}$ & Revisão sistemática da literatura. & 2014 \\
\hline
\end{tabular}

Fonte: Autores (2021).

O Quadro 2, apresenta os artigos usados no estudo, segundo sua autoria e objetivo.

Quadro 2. Artigos referentes ao estudo.

\begin{tabular}{|c|c|}
\hline Autor & bjetivo \\
\hline $\begin{array}{l}\text { Magagninet } \\
\text { al. }\end{array}$ & $\begin{array}{l}\text { Compreender os hábitos, dificuldades e as estratégias alimentares de crianças e adolescentes com } \\
\text { transtorno do espectro autista (TEA). }\end{array}$ \\
\hline $\begin{array}{l}\text { Moreira, } \\
\text { Lima } \quad \& \\
\text { Guerra. }\end{array}$ & $\begin{array}{l}\text { Avaliar a sobrecarga objetiva e subjetiva de familiares cuidadores de criança com transtorno do espectro } \\
\text { autista. }\end{array}$ \\
\hline $\begin{array}{l}\text { Portes \& } \quad \& \\
\text { Vieira. }\end{array}$ & $\begin{array}{l}\text { Compreender a percepção de pais e mães com filhos diagnosticados com transtorno do espectro autista, de } \\
\text { sua relação coparental. }\end{array}$ \\
\hline $\begin{array}{l}\text { Bagaioloet } \\
\text { al. }\end{array}$ & $\begin{array}{l}\text { Capacitar, com base na Análise Aplicada do Comportamento, cuidadores } \\
\text { de indivíduos com TEA que apresentam comportamento disruptivo e deficitde comunicação. }\end{array}$ \\
\hline $\begin{array}{l}\text { Fonseca et } \\
\text { al. }\end{array}$ & Identificar as influências do TEA nas relações familiares. \\
\hline $\begin{array}{l}\text { Balestro\& } \\
\text { Fernandes. }\end{array}$ & $\begin{array}{l}\text { Analisar a percepção de cuidadores de crianças com Transtorno do Espectro do Autismo quanto ao perfil } \\
\text { funcional da comunicação de seus filhos em três momentos, antes e após as orientações. }\end{array}$ \\
\hline $\begin{array}{ll}\text { Corrêa } \& \\
\text { Queiroz. }\end{array}$ & $\begin{array}{l}\text { Analisar as trocas sociais entre crianças com diagnóstico de TEA, submetidas a um programa de } \\
\text { intervenção precoce, e suas mães, também participantes do programa. }\end{array}$ \\
\hline $\begin{array}{l}\text { Tabaquimet } \\
\text { al. }\end{array}$ & $\begin{array}{l}\text { Identificar a relação do padrão de independência da criança com TEA e o nível de autoeficácia do seu } \\
\text { cuidador. }\end{array}$ \\
\hline Gomes et al. & $\begin{array}{l}\text { Descrever os desafios encontrados pelas famílias na convivência com crianças portadoras de transtorno do } \\
\text { espectro autista (TEA) no Brasil e as estratégias de superação empregadas. }\end{array}$ \\
\hline
\end{tabular}

Fonte: Autores (2021).

Segundo Moreira, Lima e Guerra (2020), as crianças com Transtorno do Espectro autista (TEA), dependem em alto grau da presença de um cuidador no âmbito domiciliar, para realização das atividades do cotidiano, e uma maneira conceituada como continua, e assim caracterizando o cuidador domiciliar como fator essencial para a promoção de bem estar ao paciente autista, e por se caracterizar como atividade continua, o cuidador sofre desgaste emocional e físico, ocasionando consequências 
como falta de tempo para desempenhar tarefas relacionadas a sua própria vida, estresse, problemas de saúde mental e a necessidade de adequar a própria vida para suprir as necessidades do ser cuidado.

Analisando os estudos de Tabaquimet al (2015), a distribuição das tarefas dentro do domicílio é quase exclusivamente delegado aos cuidadores do sexo feminino. Sendo reforçado pelos estudos de Portes e Vieira (2020), no qual aponta que as cuidadoras caracterizam as atividades de seus parceiros como algo auxiliar, desempenhando atividades de lazer, como brincar e cantar, assim as mães de crianças autistas se caracterizam como principais cuidadoras, promovendo cuidados básicos de vida, como dar banho, comida e garantir que a criança visite o ambiente hospitalar para atendimento com a equipe multiprofissional de saúde.

O desequilíbrio na divisão do cuidado gera a cuidadora um sentimento de sobrecarga e exaustam, na qual o membro masculino colabora pouco para as atividades diárias de cuidado, e assim destacando que a grande maioria das cuidadoras são donas de casa, e consorciam o cuidado ao paciente autista com as atividades domésticas (Portes \& Vieira, 2020).

$\mathrm{O}$ cuidador no contexto domiciliar tem dificuldade para expor a criança com autismo a situações estressoras, como a realização de testes laboratoriais ou vacinação, sugerindo que existe um sentimento se superproteção por parte dos cuidadores, e ocasionando como consequência o não convívio do paciente com pessoas fora do círculo familiar (Tabaquimet al, 2015).

Segundo Bagaiolo (2019), a complexidade do ato de cuidar é algo que está em constante presença na vida dos cuidadores, enfatizando a importância e necessidade de se trabalhar com capacitação desses familiares, na qual se identificou vários fatores que dificultam essa atividade, sendo eles a falta de adesão da família aos serviços promotores de capacitação, pouco apoio familiar, dificuldade em perceber a necessidade de melhorar a qualidade do serviço prestado e problemas financeiros.

O convívio com o paciente portador de TEA coloca a família em situações ainda não vivenciadas, no qual se consolida como desafio e muda toda a rotina dos familiares, e assim surge a necessidade dos serviços de promoção de saúde e visitas domiciliares, na qual são oferecidos espaços de acolhimento, diálogos e orientações de saúde, para que as atividades desempenhadas no contexto domiciliar sejam mais efetivas, e assim tornando-se essencial a participação da equipe de saúde no cuidado a pacientes autistas (Gomes et al, 2014).

Conforme Fonseca et al (2019), é importante destacar que a família possui suas próprias características singulares, e refletindo sobre essa perspectiva, observa-se que a equipe de saúde possui a responsabilidade de através das visitas domiciliares e consultas, entender a funcionabilidade e organização familiar, afim de traças um plano de cuidados efetivo para a família aplicar ao paciente autista, desempenhando estratégias de enfrentamento as situações estressoras advindas do cuidado continuo. Em conformidade com os estudos de Corrêa e Queiroz (2017), pontua a geração de vários benefícios ao paciente, dentre eles; desenvolvimento de autoconsciência, e por consequência o desenvolvimento integral do paciente.

O suporte oferecido pelo Sistema Único de Saúde (SUS), é de fundamental importância, fornecendo profissionais de qualidade para suprir todas as necessidades do paciente, e realizando assim uma articulação entre redes de serviços, e assim consolidando na atenção primaria a saúde, a base para o desenvolvimento do indivíduo portador de TEA (Magagninet $a l, 2021)$.

Um dos limites desse estudo, foi a pouca quantidade de artigos na integra, que abordassem com objetividade as ações dos cuidadores desenvolvidas no contexto familiar, enfatizando o cuidado continuo a pessoas portadoras de TEA, e assim foi necessário a elaboração de novos estudos que abordem enfaticamente a ação desses cuidadores no contexto do domicilio.

\section{Conclusão}

Esse estudo é relevante a maneira que possibilita conhecer as atividades desempenhadas pelos cuidadores familiares ao paciente portador do Transtorno do Espectro Autista, e assim conhecer as dificuldades encontradas na implementação 
dessas atividades de cuidado. Com o conhecimento adquirido através da pesquisa nas bases de dados, observa-se a importância que o cuidador exerce para a promoção da qualidade de vida ao paciente, e como é a articulação da equipe de saúde para realizar a capacitação dessas famílias.

Enfatiza-se a necessidade de promover saúde a esses cuidadores, pois ficou claro durante a construção do estudo, que essa comunidade ainda é carente de informações sobre o diagnóstico e tratamento de TEA.

Essa pesquisa influencia a produção de novos estudos dentro dessa temática, que possa corroborar para uma melhor assistência domiciliar ao paciente autista no futuro.

\section{Referências}

Balestro, J., \& Fernandes, F. (2019). Percepção de cuidadores de crianças com Transtorno do Espectro do Autismo quanto ao perfil comunicativo de seus filhos após um programa de orientação fonoaudiológica. Codas, 31(1). 10.1590/2317-1782/20182018222.

Bagaiolo, L., Bordini, D., Cunha, G., Sasaki, T., Nogueira, M., Pacífico, C., \& Braido, M. (2019). Implementing a community-basedparent training behavioralintervention for Autism Spectrum Disorder. Psicologia - Teoria E Prática, 21(3). 10.5935/1980-6906/psicologia.v21n3p456-472.

Balestro, J., \& Fernandes, F. (2019). Percepção de cuidadores de crianças com Transtorno do Espectro do Autismo quanto ao perfil comunicativo de seus filhos após um programa de orientação fonoaudiológica. Codas, 31(1). 10.1590/2317-1782/20182018222.

Corrêa, M., \& Queiroz, S. (2017). A família é o melhor recurso da criança: análise das trocas sociais entre mães e crianças com transtorno do espectro do autismo. Ciências \& Cognição, 22(041-062), 44- 62.

Ercole, F., Melo, L., \& Alcoforado, C. (2014). Integrative review versus systematic review. Reme: Revista Mineira De Enfermagem, 18(1). 10.5935/14152762.20140001.

Fonseca, L., Marques, I., Mattos, M., \& Gomes, D. (2019). Influências Do Transtorno Do Espectro Autista Nas Relações Familiares: Revisão Sistemática. Revista Baiana De Saúde Pública, 43(2), 444-465.

Gomes, P., Lima, L., Bueno, M., Araújo, L., \& Souza, N. (2015). Autism in Brazil: a systematic review offamilychallengesandcopingstrategies. Jornal De Pediatria, 91(2), 111-121. 10.1016/j.jped.2014.08.009.

Brasil, M. (2021). Diretrizes de Atenção à Reabilitação da Pessoa com Transtornos do Espectro do Autismo (TEA) (1st ed., pp. 5 - 84). Brasília: Secretaria de Atenção à Saúde.

Magagnin, T., Silva, M., Nunes, R., Ferraz, F., \&Soratto, J. (2021). Aspectos alimentares e nutricionais de crianças e adolescentes com transtorno do espectro autista. Physis: Revista De Saúde Coletiva, 31(1). 10.1590/s0103-73312021310104.

Organização pan-americana de Saúde. Organização Mundial da Saúde (org.). Transtorno do espectro autista. 2020. Disponível em: https://www.paho.org/pt/topicos/transtorno-do-espectro-autista. Acesso em: 13 jul. 2021.

Maciel Portes, J., \& Vieira, M. (2020). Coparentalidade No Contexto Familiar De Crianças Com Transtorno Do Espectro Autista. Psicologia Em Estudo, 25. 10.4025/psicolestud.v25i0.44897.

Soares, W. (2021). Um retrato do autismo no Brasil. http://www.usp.br/espacoaberto/?materia=um-retrato-do-autismo-no-brasil.

Tabaquim, M. de L. M., et al. (2015). Autoeficácia de cuidadores de crianças com o transtorno do espectro autista. Revista Psicopedagogia, 32(99), 285-292. http://pepsic.bvsalud.org/scielo.php?script=sci_arttext\&pid=S0103-84862015000300002\&lng=pt\&tlng=pt. 